\title{
French Seventeenth-Century Literature. Influences and transformations. Essays in honour of Christopher J. Gossip, Jane Southwood, Bernard Bourque eds.
}

\section{Chiara Mainardi}

\section{(2) OpenEdition}

\section{Journals}

\section{Edizione digitale}

URL: http://journals.openedition.org/studifrancesi/5933

DOI: $10.4000 /$ studifrancesi.5933

ISSN: 2421-5856

\section{Editore}

Rosenberg \& Sellier

\section{Edizione cartacea}

Data di pubblicazione: 1 mai 2011

Paginazione: 165-166

ISSN: 0039-2944

\section{Notizia bibliografica digitale}

Chiara Mainardi, «French Seventeenth-Century Literature. Influences and transformations. Essays in honour of Christopher J. Gossip, Jane Southwood, Bernard Bourque eds.», Studi Francesi [Online], 163 (LV | I) | 2011, online dal 30 novembre 2015, consultato il 13 janvier 2021. URL: http:// journals.openedition.org/studifrancesi/5933 ; DOI: https://doi.org/10.4000/studifrancesi.5933

Questo documento è stato generato automaticamente il 13 janvier 2021.

\section{cc) (†)}

Studi Francesi è distribuita con Licenza Creative Commons Attribuzione - Non commerciale - Non opere derivate 4.0 Internazionale. 


\title{
French Seventeenth-Century Literature. Influences and transformations. Essays in honour of Christopher J. Gossip, Jane Southwood, Bernard Bourque eds.
}

\author{
Chiara Mainardi
}

\section{NOTIZIA}

French Seventeenth-Century Literature. Influences and transformations. Essays in honour of Christopher J. Gossip, Jane SOUTHWOOD, Bernard BOURQUE eds., Bern, Peter Lang, 2009, pp. XVII-275.

1 Omaggio al celebre studioso Christopher J. Gossip, professore emerito all'Università di New England, Australia, questo volume racchiude tredici saggi sul Seicento francese. Essi affondano le radici in una delle aree di ricerca predilette dallo studioso e si diramano in tre linee principali: la ripresa di temi antichi nella letteratura francese del Seicento, il riadattamento di soggetti identici da parte di autori diversi e l'influenza che alcuni scrittori del diciassettesimo secolo ebbero nelle epoche seguenti. Le ricerche, redatte in lingua inglese e francese, sono disposte secondo l'ordine alfabetico degli autori: nel darne conto, le raggrupperemo invece in base all'argomento trattato.

Il primo saggio è affidato alla penna di H. T. BARN-WELL, Metamorphoses of a Dramatic Narrative: The Dea-th of Hippolytus from Euripides to Racine (pp. 1-20). Barnwell, partendo dall'analisi delle fonti classiche, la Fedra di Euripide e quella di Seneca, analizza le rielaborazioni del mito di Ippolito effettuate da Garnier e da La Pinelière, nonché da Gilbert, Bidar e Pradon, per giungere al celebre Phèdre et Hippolyte di Racine.

3 Anche l'articolo di D. F. conNon, Animal Intincts: Homer, Plutarch and La Fontaine go to the Fair (pp. 75-90) s'interessa all'antichità, passando dapprima in rassegna Omero, Plutarco, Ovidio, Dante e il fiorentino Giovan Battista Gelli, per poi chiarire quali sono 
state le trasformazioni adottate dagli autori appena citati rispetto al decimo libro dell'Odissea, relativo all'incontro tra Ulisse e la maga Circe. Infine l'autore dimostra in che modo queste modificazioni vennero riprese sia da La Fontaine, per caratterizzare gli animali delle sue favole, che da L. Fuzelier e M.C. Le Grand per la loro pièce Les animaux raisonnables (1718).

4 Al centro della ricerca di W. BRоoкs Philippe Quinault, Thomas Corneille, and Contrasting Approaches to Common Dramatic Material: "Stratonice" and "Antiochus" (pp.21-37), troviamo l'analisi di due autori fra loro contemporanei che si cimentano con lo stesso tema. L'autore illustra le somiglianze e le differenze tra la tragedia Antiochus di Thomas Corneille e la tragicommedia Stratonice di Quinault non solo per quanto riguarda la struttura interna delle opere, ma anche per ciò che concerne l'atteggiamento dei vari personaggi, per illustrare i motivi che generarono la redazione di queste due opere.

Il saggio di B. J. BOURQUe Deux Versions de "Zénobie": imitation ou trasformation? (pp. 219-233) mostra come la tragedia dell'abbé d'Aubignac venne ripresa, a soli dodici anni di distanza dalla sua pubblicazione, da Jean de Magnon. L'articolo si propone di spiegare quali sono le variazioni rispetto al lavoro di d'Aubignac, approfondendo sia la struttura dell'opera che gli elementi relativi al soggetto storico, ai personaggi, alle unità aristoteliche e agli aspetti eterogenei presenti nelle varie scene.

6 Un altro gruppo, composto da cinque articoli, affronta la questione del riadattamento di opere del Seicento francese in romanzi, opere teatrali o film durante il xx e XXI secolo. Il primo contributo è quello di L. BROTHERSON, Religion in a Quebec Novel: A Catholic Heritage Transformed (pp.39-55), dove si esamina il romanzo di Gabrielle Roy, Bonheur d'occasion (1945). L'A. mette in evidenza come questa religione, ereditata dai missionari francesi che colonizzarono il Québec nel XVII secolo, sia stata indebolita da vari fattori, come l'urbanizzazione e il consumismo, che ne hanno svigorito le pratiche. Considerando il cattolicesimo come caratteristica centrale della società francese del Seicento, l'A. mostra come essa continui a risuonare sia nella psiche dei personaggi del romanzo quebecchese contemporaneo che nel modo in cui essi si relazionano gli uni con gli altri.

7 H. DUfFY, nel suo contributo L'(In)fidélité? A Kristevan Reading of Andrzej 'Zuławski's Cinematic Adaptation of "La Princesse de Clèves" (pp. 91-115), commenta il film La fidelité di Andrzej 'Zuławski, tratto dal romanzo La Princesse de Clèves di Mme de La Fayette, secondo le teorie di Julia Kristeva. Le conseguenze provocate dal lutto irrisolto per la perdita della madre sulla psiche tormentata della protagonista sono pregnanti per lo sviluppo dell'articolo. Riallacciandosi ai concetti psicanalitici della depressione e della malinconia femminile, l'autrice espone la sua personale interpretazione del film di 'Zuławski.

8 J. HATTE illustra nel saggio Truth, Lies and Time-Travel: Jean Cocteau in the "Impromptu" Tradition (pp.117-138) come Cocteau, negli anni che vanno dal 1937 al 1962, si sia avvalso della forma teatrale libera detta impromptu per scrivere cinque delle sue opere. L'autrice, dopo averne analizzato le basi, il significato, le origini secentesche e il suo utilizzo durante i secoli, si concentra su due testi in particolare, La Maison hantée e L'Impromptu du Palais-Royal.

9 Segue Jean-Marie Villégier's Molière: Experiments in Theatrical Morphogenesis (pp. 139-155) dove N. PЕACOCK articola la sua riflessione sulle modalità che riguardano il rifacimento delle opere di Molière da parte di Villégier. Specializzato nel repertorio francese del 
Seicento, Villégier interpreta le opere di Molière in chiave moderna. Il regista, anche in collaborazione col direttore d'orchestra William Christie, rileva gli aspetti particolari che emergono da ciascun testo e mantiene la forza vitale che distingue ogni opera.

L'articolo di J. SOUTHWOOD, Éléments antiques et classiques dans l'œuvre de Marguerite Yourcenar: "Le Coup de grace" (pp. 235-259) si sofferma dapprima sulla predilezione della Yourcenar per la Grecia e la Roma del passato, poi sulla contiguità che intercorre tra l'antichità e il presente, utilizzando i miti greco-romani per mettere in evidenza l'universalità della natura umana. Giunge poi ad analizzare due opere yourcenariane, Feaux e Le Coup de grâce che Southwood illustra in base alla tematica dell'amore tragico, della tragedia classica, delle unità aristoteliche e delle bienséances. Entrambe le opere sono collegabili alla tragedia del xvII secolo e sono legate dal grande tema tragico dell'amore.

11 Con l'articolo di J. CAMPBell The Metamorphoses of Innocence in Racine's "Esther" (pp. 57-73), l'attenzione si concentra su un solo autore secentesco, in questo caso Racine. La tragedia che l'A. prende in considerazione in questo articolo, Esther, ha una struttura prettamente drammatica nonostante si basi su di un tema biblico. L'innocenza della protagonista, sebbene già presente nel testo sacro, subisce nella trasposizione teatrale sensibili variazioni che vengono analizzate anche in relazione a fattori legati alla rappresentazione scenica.

H. PHILLIPS con il suo contributo Scenes in the City: Urban Developments in SeventeenthCentury French Theatre (pp.157-174) si propone di analizzare le trasformazioni della città di Parigi dovute allo sviluppo urbano del diciassettesimo secolo, e il modo in cui queste si riflettono in molte opere del periodo. Le commedie del Seicento francese incorporano in modo esemplare non solo gli sviluppi urbani della città di quel periodo, ma altresì le caratteristiche della vita cittadina. Mentre i primi sono peculiari di una singola città e definiscono l'individualità della stessa, le seconde sono più generali, creano uno spazio globale, senza confini, che permette di trasferire l'azione da un luogo all'altro e di unire gli spettatori in uno spazio universale.

13 M. S. RIVIÈRE nel suo saggio Prussian Princesses at the Court of Frederick II: Devotees of the Theatre of Racine and Voltaire (pp. 175-197) affronta invece come viene recepito il teatro di Racine e di Voltaire da parte della società prussiana del xviII secolo. L'A. esamina soprattutto come Voltaire orchestrò un'intelligente campagna pubblicitaria in tutta Europa, e in Prussia in particolare, promuovendo, unitamente all'opera raciniana, anche la sua propria. Attraverso le visite alla corte di Federico II e la fitta corrispondenza con le principesse tedesche, Voltaire riuscì a procurare importanti benefici alla sua professione. La sua carriera, rafforzata dalla passione delle principesse per le sue opere, veniva infatti promossa nei circoli letterari; le molte ammiratrici di sesso femminile erano spettatrici avide di tragedie e, pur continuando ad apprezzare il teatro raciniano, incentivavano la pubblicazione e il successo dei lavori di Voltaire.

Infine, A. SAUNDERS con l'articolo Who can put on a better show?: Celebrations of Peace as Mounted in Jesuit Colleges in Paris and Lyon in 1660 (pp. 199-217) propone i risultati di una ricerca personale sui festeggiamenti di Parigi e Lione in riferimento al Trattato dei Pirenei, e il conseguente matrimonio reale tra il giovane Luigi XIV e l'Infanta di Spagna del 1660. L'autrice analizza e approfondisce non soltanto le celebrazioni che avvennero nelle due città, ma anche nei rispettivi collegi dei gesuiti, il Collège de la Trinité di Lione e quello di Clermont a Parigi, in modo da offrire una visione d'insieme e stabilire 
quale fosse il legame tra le due città francesi e le rispettive scuole nella seconda metà del XVII secolo.

15 Alcune notizie bibliografiche sugli autori e sui collaboratori concludono il volume. 